\title{
NANOINDENTATION AND ACCELERATED PROPERTY MAPPING OF PORTLAND CEMENT PASTE
}

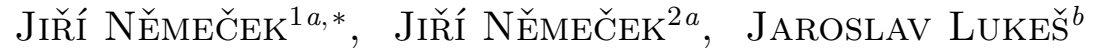 \\ ${ }^{a}$ Czech Technical University in Prague, Faculty of Civil Engineering, Department of Mechanics, Thákurova 7, \\ 16629 Prague 6, Czech Republic \\ ${ }^{b}$ Czech Technical University in Prague, Faculty of Mechanical Engineering, Department of Mechanics, \\ Biomechanics and Mechatronics, Czech Republic \\ * corresponding author: jiri.nemecek.1@fsv.cvut.cz (Ph.D. candidate)
}

\begin{abstract}
This paper presents a new nanoindentation mode: accelerated property mapping (XPM) applied to one year old Portland cement paste. The principle of the innovative method is similar to classical quasi-static nanoindentation but the time necessary for performing one indent is significantly decreased from minutes to tenths of seconds. The XPM mode is capable of measuring maps of local mechanical properties (reduced modulus, hardness). The method was compared to classical nanoindentation mode. The main advantages of XPM measurement are higher spatial resolution, lower property fluctuation and lower acquisition time. However, XPM is not able to measure some parameters such as creep. Also, high speed loading of XPM brings high strain effect which artificially shifts values of reduced modulus to higher values compared to quasi-static loading cases.
\end{abstract}

KEYWORDS: Cement paste, high speed nanoindentation, strain rate, XPM.

\section{INTRODUCTION}

Nanoindentation is a widely used technique for micromechanical testing of materials. Its principle lies in pressing a tip with known geometry to a material. During the indentation process the loading response is measured. Classical nanoindentation performs one indent in the order of minutes which leads to long measurement time, especially if hundreds of indents needs to be performed [1]. In recent years, a new mode of testing called accelerated property mapping (XPM) has been developed by Bruker-Hysitron company. XPM presents a way to perform hundreds of indents in a few minutes and create maps of reduced modulus and hardness with very high spatial resolution. The testing itself is done by three capacitive plate transducer where the tip is attached to piezo ceramic and loading is then applied electrostatically. Classical nanoindentation (NI) measurement consists of several steps: moving tip to the position (a few seconds), piezo settlement (80-200 s), drift measurement (40-80 s) and quasi-static loading (typically a few seconds). During the XPM measurement, the piezo settlement and drift measurement are done only before indentation. So, the process of XPM measurement consists only of performing the indent and changing the position. Thus, such a process must be very quick in order to avoid changing the drift rate. Elastic modulus is then derived from the unloading curve by Oliver and Pharr theory [2]. The theory is usually implemented as a standard in nanoindenter's softwares for, both NI and XPM. However, if the ef-

\footnotetext{
${ }^{1} \mathrm{Ph}$. D. candidate at CTU in Prague

${ }^{2}$ Associate Professor at CTU in Prague
}

fect of strain rate is taken into account then it can result in an increase of mechanical properties for some materials tested by nanoindentation, i.e., metals 3, 4].

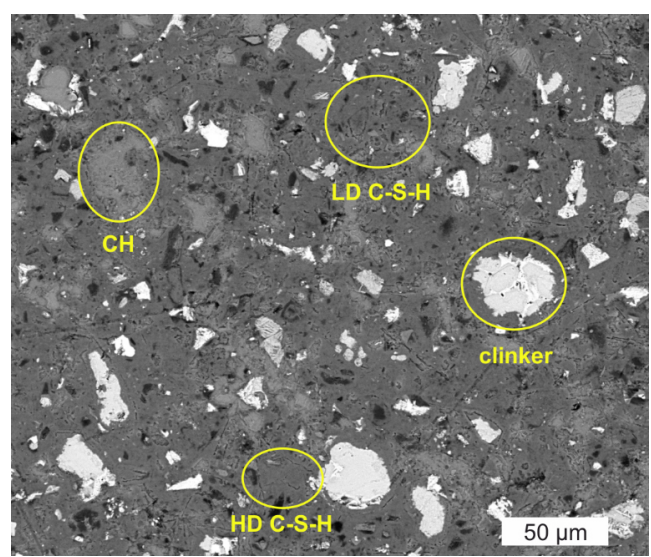

Figure 1. Typical SEM-BSE image of cement paste showing individual phases.

Our study is focused on cementitious materials where the strain-rate effect of macroscopic mechanical properties e.g. an increase of compressive strength is observable on macro-scale [5]. Similar effect can be observed on micro-scale using XPM method which affects the results and has not yet been properly studied. However, using XPM method on cementitious materials would be very advantageous. Generally, hundreds of indents are needed for the characterization of highly heterogeneous composites [6, 7] and saving time would be a huge benefit. On the micro-scale, it is possible to characterize individual chemical phases by scanning electron microscopy (SEM) and energy dispersion X- 
ray spectroscopy (EDS). Hydrated cement paste is mainly composed of Calcium-Silica-Hydrate (C-S-H gel) existing in two characteristic densities, low (LD C-S-H) and high (HD C-S-H), crystalline calcium hydroxide $(\mathrm{CH})$, residual clinker and porosity [7]. The typical layout of the phases is shown in Fig. 1 .

This study aims to compare both methods on a cementitious composite, highlight their advantages and quantify the strain-rate effect of XPM method with rate dependent visco-plastic constitutive model.

\section{MATERIALS AND METHODS}

\subsection{SAMPLE PREPARATION}

A hydrated cement paste from Portland cement CEM I 42.5R (from Mokrá, Czech Republic) was prepared for testing purposes. Cement was mixed with water to cement ratio 0.4 , cast into cylindrical molds with height of $32 \mathrm{~mm}$ and diameter of $20 \mathrm{~mm}$. The samples were demoulded after three days and stored in the water. 28 days old samples were then cut into $5 \mathrm{~mm}$ thick slices on precise cut off machine and dried at $50{ }^{\circ} \mathrm{C}$ for one day. The surface of the samples was then grinded with $\mathrm{SiC}$ papers up to grit size of 4000. Finally, samples were put into ethanol into ultrasonic cleaner to remove all loose particles.

\subsection{NANOINDENTATION}

The standard quasi-static nanoindentation test was performed using Hysitron TI-700 with Berkovich diamond tip. Seven grids of 10x10 indents with separation of $12.5 \mu \mathrm{m}$ were prescribed to cover the heterogeneity of the sample. Trapezoidal loading diagram with linear loading lasting 3 seconds up to $2 \mathrm{mN}$, then by 20 seconds holding period, followed by 3 seconds un-loading were prescribed. XPM measurement was performed on Hysitron TI-950. Maximum load and the separation between indents were carefully chosen to neglect the influence of individual indents. Eight grids with $21 \times 21$ indents with separation of $2 \mu \mathrm{m}$ were prescribed on the sample. The relatively small area of $42 \times 42 \mu \mathrm{m}$ was covered. Thus, each map was prescribed next to the residual clinker to include this less present phase into the results. The testing was force controlled with linear loading lasting $0.1 \mathrm{~s}$ up to the maximum force of $1.2 \mathrm{mN}$, followed by holding and un-loading period both lasting also $0.1 \mathrm{~s}$.

\subsection{STANDARD EVALUATION}

Reduced modulus for both testing methods (Grid NI and XPM) was evaluated from load - displacement curves using the Oliver and Pharr method [2]. The method is based on Sneddon solution [8] of a rigid indenter action on an elastic half space. The indentation modulus $E_{r}$ is defined as

$$
E_{r}=\frac{S \sqrt{\pi}}{2 \beta \sqrt{A_{c}}},
$$

where $S$ is the elastic unloading stiffness, $P_{\max }$ is the maximum load, $\beta$ is coefficient which considers different geometry of the indentation tip $(\beta=1.034$ for Berkovich tip) and $A_{c}$ is the projected contact area. Reduced modulus represents a combination of elastic stiffness of the sample and the tip. Modulus of elasticity $E$, of the sample is given by

$$
\frac{1}{E_{r}}=\frac{1-\nu^{2}}{E}+\frac{1-\nu_{i}^{2}}{E_{i}},
$$

where $E_{i}$ and $\nu_{i}$ are Young's modulus and Poisson's ratio of the indentation tip (for the diamond tip $E_{i}=1141 \mathrm{GPa}$ and $\left.\nu_{i}=0.07\right), \nu$ is the Poisson's ratio of the sample (for the cement paste $\nu=0.2$ ).

During holding period it is possible to evaluate short term creep of the material. Creep indentation parameter $C I T$ is expressed by equation

$$
\operatorname{CIT}_{\left(P, t_{1}, t_{2}\right)}=\frac{h_{2}-h_{1}}{h_{1}} \times 100,
$$

which is defined as the relative change of indentation depths $h_{1}$ and $h_{2}$ determined between the start and the end holding period at times $t_{1}$ and $t_{2}$, respectively. The parameter is dependent on contact force $P_{\max }$ and the duration of holding period.

\section{Results And Discussion}

\subsection{STANDARD NANOINDENTATION}

The results of NI gives Young's modulus and hardness maps (Fig. 2a) with relatively low spatial resolution. But it still gives valuable information of mechanical properties of individual phases. The range of $E$ values from 14 to $120 \mathrm{GPa}$ and $H$ from 0.4 to $14 \mathrm{GPa}$ were obtained. The frequency density plot of $E$ was constructed from 700 indents, see Fig 3. The values of $E$ corresponding to the main peak lie in the range of 23-38 $\mathrm{GPa}$ and are mostly composed with LD C-S-H and HD C-S-H gels, the peak of $\mathrm{CH}$ was found around $42 \mathrm{GPa}$ and the rest of the indents with Young's modulus higher than $50 \mathrm{GPa}$ belong to residual clinker. These results correspond well with literature 6, 7]. The total time needed for performing one grid of indents (100 indents) was around $500 \mathrm{~min}$ utes.

\subsection{ACCELERATED PROPERTY MAPPING}

Eight maps of mechanical properties were obtained by XPM method. Totally 3548 indents were done with very high spatial resolution of $2 \mu \mathrm{m}$. The time needed for acquiring one map was about 5 minutes. An example of measured areas by XPM is shown in Fig. 2b. Young's modulus and hardness maps corresponds well with SEM-BSE image. Thus, it suits well for identification of individual phases and their boundaries. Also, due to high spatial resolution, XPM is capable of identifying individual clinker minerals, red and orange color in Fig. $2 \mathrm{~b}$ with values of $E$ in range of 115-181 GPa. The moduli of other phases were identified as: LD C-S-H $(E=30-32 \mathrm{GPa})$, HD C-S-H $(E=40-46 \mathrm{GPa}), \mathrm{CH}(E=50-56 \mathrm{GPa})$. These 


\section{SEM-BSE}
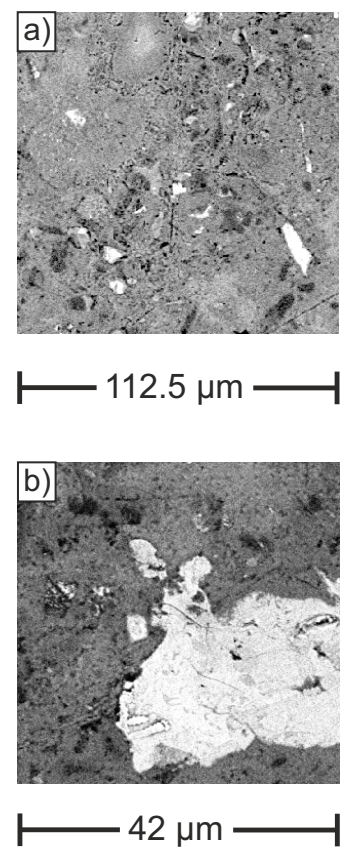

Young's modulus, $E[\mathrm{GPa}]$ Hardness, $H[\mathrm{GPa}]$
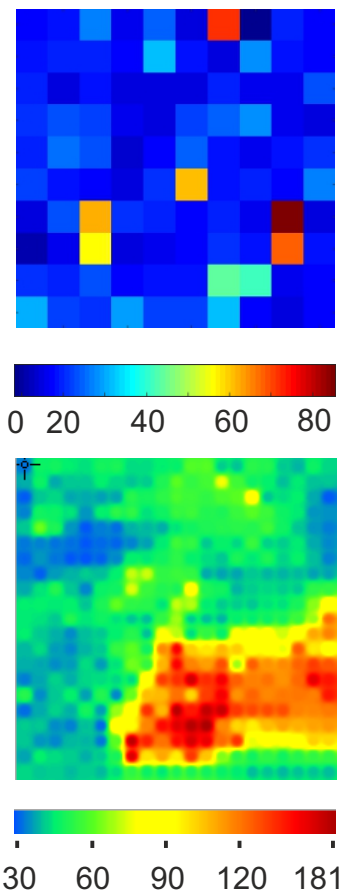
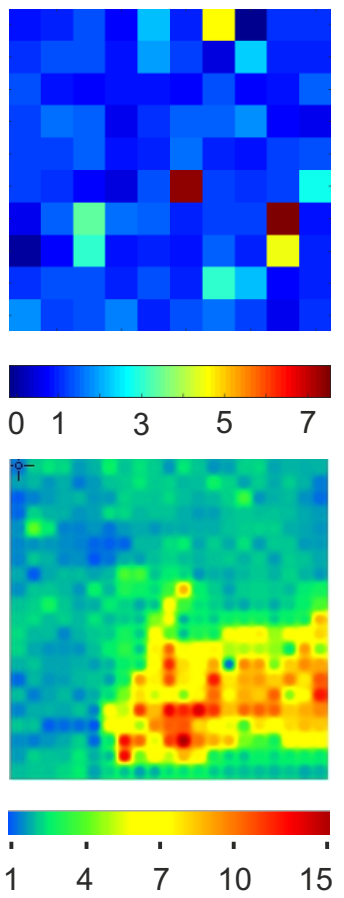

Figure 2. SEM-BSE image, Young's modulus, Hardness maps measured by a) NI b) XPM.

values are higher than the literature results [6, 7]. However, the results correspond well with XPM study of Sebastiani et al. [9]. Again, the frequency plot of $E$ was constructed, see Fig 3 .

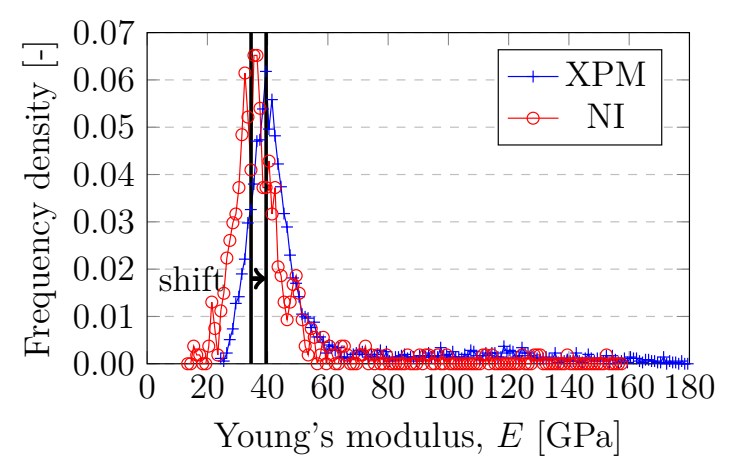

Figure 3. Frequency plots of NI and XPM with highlighted shift of the main peak.

\subsection{INDENTATION CREEP PARAMETER}

$C I T$ was evaluated from 20 second holding period of NI technique. The results of individual phases are shown in Table 1 and a different parameter was found for each individual phase. The highest value was found for C-S-H gel around 9-12\%. Also, some deformation was found for $\mathrm{CH}$ and residual clinker, where it is well known that these phases do not creep. However, during indentation hard particles of clinker are pressed into surrounding matrix of hydrates, which affects the results. Again, CIT parameter was evaluated from the holding period of XPM. A very interesting fact was found though: the value of $C I T$ was $6.8 \pm 0.3 \%$

\begin{tabular}{cccc}
\hline & XPM & \multicolumn{2}{c}{$\mathrm{NI}$} \\
\hline Phase & $1.2 \mathrm{mN}$, & $2.0 \mathrm{mN}$, & $2.0 \mathrm{mN}$, \\
& $0.1 \mathrm{~s}, 0.2 \mathrm{~s}$ & $3.0 \mathrm{~s}, 3.1 \mathrm{~s}$ & $3.0 \mathrm{~s}, 23.0 \mathrm{~s}$ \\
LD C-S-H & $6.8 \pm 0.3 \%$ & $1.2 \pm 0.3 \%$ & $11.3 \pm 3.1 \%$ \\
HD C-S-H & $6.8 \pm 0.3 \%$ & $1.1 \pm 0.3 \%$ & $9.5 \pm 3.3 \%$ \\
CH & $6.8 \pm 0.3 \%$ & $0.8 \pm 0.2 \%$ & $6.4 \pm 3.7 \%$ \\
Clinker & $5.9 \pm 0.6 \%$ & $0.7 \pm 0.2 \%$ & $2.1 \pm 1.9 \%$ \\
\hline
\end{tabular}

TABlE 1. Creep indentation parameter CIT calculated in different phases for XPM and NI.

regardless of the indentation phase. However, different indentation forces were used. Thus, the values are not directly comparable for XPM and NI. The CIT was evaluated for $0.1 \mathrm{~s}$ holding period from NI. Smaller values with different values were found, even if higher force was used. Such findings suggest that during XPM, creep of the material is not correctly measured and the results might be affected by creep of the piezo scanner.

\subsection{MUtUAL COMPARISON}

Grid NI brings very low spatial resolution with very good absolute values of mechanical properties of LD, HD C-S-H gel and $\mathrm{CH}$. The CIT parameter provides comparison of creep deformation between individual phases. XPM gives higher spatial resolution somewhere close to SEM-EDS maps. The area covered by individual maps is lower than that for NI. However, time needed for obtaining one map is significantly decreased from hours to minutes. The creep parameter 

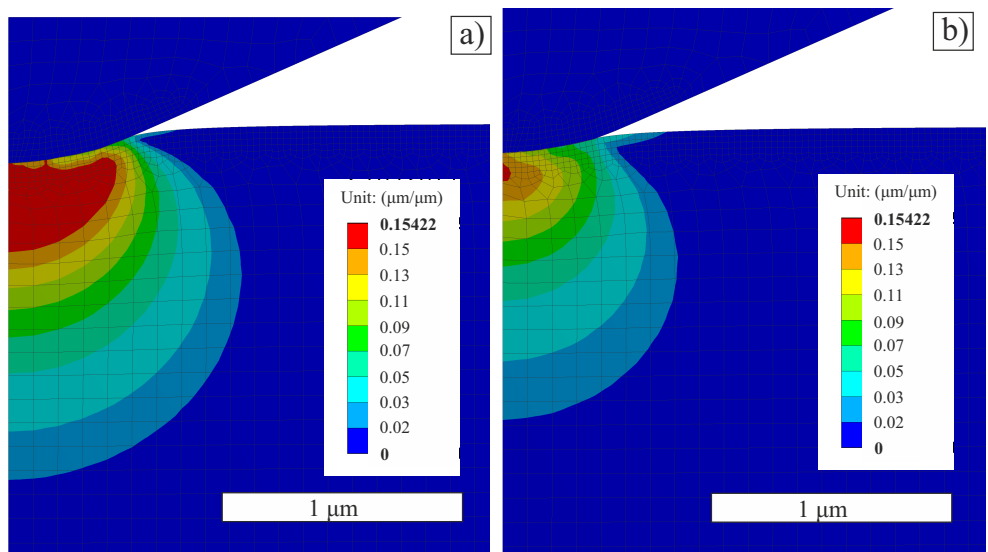

Figure 4. Equivalent von Mises strain under the tip at the end of loading a) at time $=3 \mathrm{~s}, \mathrm{speed}=0.67 \mathrm{mN} / \mathrm{s}(\mathrm{NI})$ b) at time $=0.1 \mathrm{~s}$, speed $=20 \mathrm{mN} / \mathrm{s}(\mathrm{XPM})$.

of material is not obtained from the method. Quantitatively, XPM provides data comparable to NI but slightly shifted towards higher values. The shift of $14.8 \%$ was calculated as the ratio of Young's modulus of the points with the highest frequency density in the histogram measured by NI and XPM, respectively (Fig. 3). Such a deviation could be explained by strain-rate effect and was further investigated with numerical modeling.

\section{NumericAl MODELING}

The strain rate effect was studied by finite element method (FEM) in ANSYS software as 2D asymmetrical task with transient dynamic solver. The real shape diamond indenter (Elastic isotropic: $E_{i}=1141 \mathrm{GPa}$ and $\left.\nu_{i}=0.07\right)$ was brought to contact with an elastoplastic domain with size of $15 \times 15 \mu \mathrm{m}$ of the cement sample. Loading rate effects were captured by viscoplastic Perzyna model [10. The model calculates equivalent plastic strain as

$$
\dot{\varepsilon}_{p l}=\gamma\left(\frac{\sigma}{\sigma_{0}}-1\right)^{1 / m},
$$

where $\gamma$ is the material viscosity parameter, $\sigma$ is the effective stress, $m$ is the strain rate hardening coefficient, $\sigma_{0}$ is the static yield point of the elasto-plastic model. Two different nanoindentation machines were used and the tip geometry plays an important role during the fitting load-displacement data. Thus, the parameters were identified only to best-fit the experimental NI curve $($ speed $=0.67 \mathrm{mN} / \mathrm{s})$. The parameters were identify as: $m=0.8, \gamma=0.025$, density $=$ $2000 \mathrm{~kg} / \mathrm{m}^{3}, E=33.8 \mathrm{GPa}, \nu=0.2, \sigma_{0}=300 \mathrm{MPa}$, tangent modulus $=2000 \mathrm{MPa}$. After parameters were identified, the same set of parameters was used for XPM loading $($ speed $=20.0 \mathrm{mN} / \mathrm{s})$. The results of fitting are shown in Fig. 5

The vonMises equivalent strain under the tip was analyzed at the end of loading period, see Fig 4. It was found the during fast loading, smaller penetration depths and smaller strain are reached compared to NI but shorter loading time is leading to higher

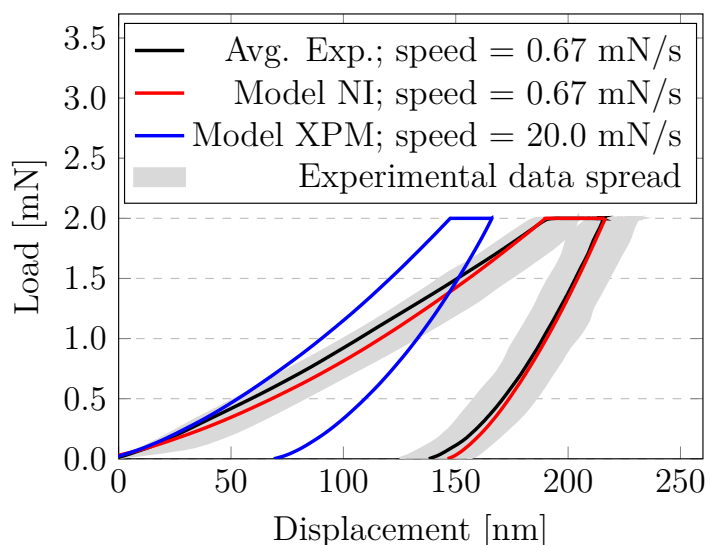

Figure 5. Calculated responses of NI and XPM loading with rate dependent viscoplastic model.

strain-rate for XPM method. The results are the following: vonMises strain $=0.1542(\mathrm{XPM})$ and 0.2009 $(\mathrm{NI})$, strain rate $=1.542 \mathrm{~s}^{-1}(\mathrm{XPM})$ and $0.067 \mathrm{~s}^{-1}$ (NI). From the above, it is obvious that XPM loading leads to $23 \mathrm{x}$ higher strain rate comprared to NI. To quantify the effect of strain rate the Oliver and Pharr theory 2 was applied on model responses. It was found that $E$ increased by $10 \%$ for XPM mode. Both experiment and numerical simulation show the shift of $E$ to higher values. The small difference between numerical modeling and experiment could be given by overlaps of individual phases within the main peak.

\section{Conclusions}

This study shows mechanical characterization of Portland cement paste with two nanoindentation testing modes: classical quasi-static grid nanoindentation (NI) and accelerated property mapping (XPM). Both techniques provide qualitatively comparable results of elastic modulus and hardness. Standard grid nanoindentation is favorable for covering larger areas and measuring creep parameters. XPM provides very fast acquisition time of hundreds indents in minutes with very high spatial resolution. The method is very good 
in identifying individual phases in a single measured map. Also, XPM artificially shifts results to higher values due to the strain rate effect. This shift was quantified as $14.8 \%$ from the experiment and calculated as $10 \%$ according to numerical simulation. In this context, the Oliver and Pharr theory which is implemented as standard in nanoindenter's softwares should be used with caution when strain-rate sensitive materials are tested with XPM technique.

\section{ACKNOWLEDGEMENTS}

Financial support of the Czech Science Foundation (project 17-05360S) and the Grant Agency of the Czech Technical University in Prague (SGS20/107/OHK1/2T/11 ) are gratefully acknowledged.

\section{REFERENCES}

[1] A. C. Fischer-Cripps. Nanoindentation. Springer, 2002. DOI:10.1007/978-0-387-22462-6

[2] W. C. Oliver, G. M. Pharr. An improved technique for determining hardness and elastic modulus using load and displacement sensing indentation experiments. Journal of materials research 7(6):1564-1583, 1992. DOI:10.1557/JMR.1992.1564

[3] E. D. Hintsala, U. Hangen, D. D. Stauffer. High-throughput nanoindentation for statistical and spatial property determination. JOM 70(4):494-503, 2018. DOI:10.1007/s11837-018-2752-0
[4] P. S. Phani, W. Oliver. Ultra high strain rate nanoindentation testing. Materials 10(6):663, 2017. DOI: $10.3390 / \mathrm{ma1} 0060663$

[5] P. H. Bischoff, S. Perry. Compressive behaviour of concrete at high strain rates. Materials and structures 24(6):425-450, 1991. DOI:10.1007/BF02472016.

[6] J. Němeček, V. Králík, J. Vondřejc. Micromechanical analysis of heterogeneous structural materials. Cement and Concrete Composites 36(0):85 - 92, 2013. DOI:10.1016/j.cemconcomp.2012.06.015

[7] G. Constantinides, F.-J. Ulm. The nanogranular nature of c-s-h. Journal of the Mechanics and Physics of Solids 55(1):64-90, 2007. DOI:10.1016/j.jmps.2006.06.003

[8] I. N. Sneddon. The relation between load and penetration in the axisymmetric boussinesq problem for a punch of arbitrary profile. International journal of engineering science 3(1):47-57, 1965. DOI:10.1016/0020-7225(65)90019-4

[9] M. Sebastiani, R. Moscatelli, F. Ridi, et al. High-resolution high-speed nanoindentation mapping of cement pastes: Unravelling the effect of microstructure on the mechanical properties of hydrated phases. Materials \& Design 97:372 - 380, 2016. DOI:10.1016/j.matdes.2016.02.087

[10] P. Perzyna. Fundamental problems in viscoplasticity. vol. 9 of Advances in Applied Mechanics, pp. 243 - 377. Elsevier, 1966. DOI:10.1016/S0065-2156(08)70009-7 